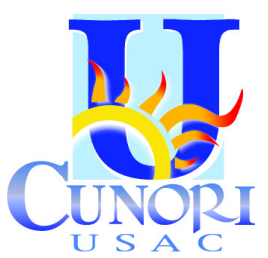

\title{
Revista Ciencia Multidisciplinaria CUNORI
}

Toxoplasma gondii: detección en carne de cerdo en la ciudad de Guatemala

(Toxoplasma gondii: detection on pork meat of Guatemala City)

Flor Dinorah Porras

Universidad de San Carlos de Guatemala

https://orcid.org/0000-0003-3368-3365

fdporras@yahoo.com

Ana Isabel Girón Samayoa

Universidad de San Carlos de Guatemala

https://orcid.org/0000-0002-6068-5836

aigironsamayoa@gmail.com

Recibido: 25/10/2020

Publicado: 19/01/2021

\section{Referencia del artículo}

Porras, F. D. y Girón Samayoa, A. I. (2021). Toxoplasma gondii: detección en carne de cerdo en la ciudad de Guatemala. Revista Ciencia Multidisciplinaria CUNORI, 5(2). 49-58.

DOI: https://doi.org/10.36314/cunori.v5i2.168

\section{Resumen}

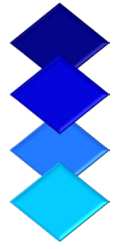

$\mathrm{O}$ BJETIVO: presentar los resultados del estudio para detección de Toxoplasma gondii en la carne de cerdo de los mercados municipales de la ciudad de Guatemala, para evaluarla como potencial fuente de infección, ya que la carne de cerdo cruda o insuficientemente cocida representa una de las fuentes de infección de Toxoplasma gondii más comunes a nivel mundial. Este protozoo es causante de una de las tres enfermedades de transmisión alimenticia más importantes. MÉTODO: se colectaron 250 muestras de carne mediante muestreo aleatorio de puestos de venta en mercados municipales de la ciudad de Guatemala, a las muestras colectadas se les realizó la prueba de qPCR. RESULTADOS: todas las muestras fueron negativas, debido a que el $68 \%$ de la carne de cerdo en los mercados municipales proviene de granjas tecnificadas que siguen medidas de bioseguridad y trazabilidad, se puede inferir que los esfuerzos de las producciones tecnificadas bajo la guía de los servicios veterinarios oficiales en bioseguridad han tenido un efecto positivo que se ve reflejado en la ausencia de $\mathrm{T}$. gondii en las muestras colectadas. CONCLUSIÓN: no se descarta la carne de cerdo como potencial fuente de infección por lo que se deben realizar más estudios en la cadena epidemiológica y de producción de toxoplasmosis en cerdos.

Palabras clave

toxoplasmosis, ETAs, salud pública, zoonosis 


\section{Abstract}

BJECTIVE: to present the results of the Toxoplasma gondii detection study in pork from the municipal markets of Guatemala city to evaluate it as a potential source of infection. Raw or insufficiently cooked pork represents one of the most common sources of Toxoplasma gondii infection at national level. world. This protozoan is the cause of one of the three most important food-borne diseases. METHOD: 250 meat samples were collected by random sampling from stalls in the municipal markets of Guatemala City. The qPCR test was performed on the collected samples. RESULTS: all samples were negative, because $68 \%$ of pork meat in municipal markets comes from technical farms that follow biosafety and traceability measures, it can be inferred that the efforts of technified productions under the guidance of the Official veterinary services have had a positive effect that is reflected in the absence of T. gondii in the collected samples. CONCLUSION: pork is not ruled out as a potential source of infection, therefore more studies should be carried out in the epidemiological and production chain of toxoplasmosis in pigs. 


\section{Introducción}

Toxoplasma gondii es un protozoo zoonótico que utiliza como hospedero definitivo a los felinos, en quienes completa un ciclo sexual hasta llegar a la liberación de ooquistes en heces. Los ooquistes desarrollan una forma esporulada resistente que puede permanecer viable e infectiva hasta por dos años, tanto en tierra como en agua. En cualquier animal de sangre caliente, estos ooquistes pueden desarrollar un ciclo asexual que culmina en la formación de quistes tisulares, los cuales son la forma infectiva para otros animales (Despommier et al., 2017).

A nivel mundial se considera a la toxoplasmosis como una de las tres enfermedades de transmisión alimenticia (ETAs) de mayor amenaza para la vida, estimando que el $50 \%$ de casos de toxoplasmosis son provocados por alimentos contaminados (Almeria y Dubey, 2020). Debido a los hábitos de consumo de carne cruda o insuficientemente cocida se le considera una de las causas más importantes dentro de las ETAs (Dubey et al., 2020).

Toxoplasmosis es la enfermedad resultante de la infección por T. gondii, su distribución es mundial, variando la prevalencia de acuerdo con la región geográfica (Sánchez-Artigas et al., 2016). Según Dubey (2010), esta prevalencia varía de un $4 \%$ en Corea del Sur hasta un $92 \%$ en Brasil. Mayoritariamente cursa como una gripe común, pero se mantiene latente en el organismo y ante inmunosupresiones puede causar desde linfadenopatías, neuropatías y uveítis, hasta lesiones nerviosas letales (Despommier et al., 2017). En su transmisión congénita, si la infección se da durante en el segundo y tercer tercio del embarazo suele ocasionar fetopatías del sistema nervioso y retinocoroiditis; mientras que durante el primer trimestre puede causar fetopatías o muerte fetal (Acha y Szyfres, 2003).

En Guatemala no hay estudios ni registros epidemiológicos sobre la presencia de la enfermedad; existen estudios aislados de seroprevalencia en grupos de interés. Gibson y Coleman (1958) encontraron seroprevalencias del $94 \%$ en 100 individuos de la costa sur del país (Escuintla) y $50 \%$ de positividad en 30 reclutas militares provenientes del altiplano, como un intento no comprobado de relación entre la prevalencia de la enfermedad y la altitud. Sinibaldi y De Ramírez (1992) hicieron distintos estudios de seroprevalencia en 550 cordones umbilicales de recién nacidos, identificando en 6 infantes la presencia de $\operatorname{lgG}$ e $\operatorname{lgM}$, lo que representa una incidencia positiva de $10.9 \%$ por cada 1000 partos. 
En 1999 se encontró una seroprevalencia de $12.4 \%$ de 532 niños de San Juan Sacatepéquez, Guatemala (Jones et al., 2005). En 2007 del total de niños seropositivos del estudio de 1999 , se encontraron $4.5 \%$ con lesiones oculares congruentes con toxoplasmosis (López et al., 2008). Por lo que, aunque no hay conocimiento de la prevalencia real de toxoplasmosis en Guatemala, existe evidencia de su presencia, siendo necesario conocer las posibles fuentes de infección para el ser humano.

Históricamente se ha considero al cerdo como una de las fuentes de infección más significativas dentro de los animales de engorde; principalmente bajo la creencia de los hábitos alimenticios omnívoros del cerdo, y para varios países se mantiene como un foco epidemiológico por la costumbre en ganadería extensiva y semi intensiva de alimentar con desechos a los cerdos (Dubey et al., 2020; Hill y Dubey, 2002; Jones y Dubey, 2012).

En Guatemala el cerdo es considerado la segunda línea de producción pecuaria, representando un consumo anual de $3.02 \mathrm{~kg}$ de carne de cerdo per cápita. Para el 2018 la población porcina nacional llegó a 798,703 individuos, que se encontraban distribuidos en un $82 \%$ producción familiar y $18 \%$ en producción comercial; dentro de la categoría comercial se contemplan dos tipos de producción (Dirección de Sanidad Animal del Viceministerio de Sanidad Agropecuaria y regulaciones del Ministerio de Agricultura, Ganadería y Alimentación [VISAR-MAGA], 2020).

Una es la granja tecnificada que cumple con los protocolos de alimentación y medidas de bioseguridad bajo el registro del Programa Nacional de Sanidad Porcina del Ministerio de Agricultura, Ganadería y Alimentación (PRONASPORC); y la segunda es la granja semi tecnificada que se caracteriza por escazas medidas de bioseguridad, permitir libre pastoreo y alimentación con desechos industriales, de mercados, restaurantes, granjas avícolas, entre otros (Dirección de Sanidad Animal VISAR-MAGA, 2020).

Ante esta realidad, donde más del $80 \%$ de producción porcina en Guatemala proviene de un manejo con pocas medidas de bioseguridad y costumbres de alimentar con desperdicios a los cerdos, manejo que aumentan el riesgo de infección con quistes tisulares, se decidió analizar la carne de los mercados municipales de la Ciudad de Guatemala como potencial fuente de infección de Toxoplasma gondii para seres humanos. 


\section{Materiales y métodos}

Se obtuvieron 250 muestras de carne de cerdo provenientes de 342 puestos de venta de carne ubicados en 23 mercados municipales de la ciudad de Guatemala. A través de la fórmula de muestro de proporciones con poblaciones finitas, utilizando $95 \%$ de confianza, 3\% de margen de error y $70 \%$ de frecuencia esperada, que fue tomada de la prevalencia encontrada en el estudio de Lora y colaboradores (2007) que proviene de una región con similares características. A las muestras de carne colectadas se les extrajo el ADN mediante un método de extracción de columnas utilizando el kit comercial High Pure PCR Template Preparation Kit@. AI ADN extraído se le realizó la técnica qPCR, con los primer utilizados amplifican una región del gen B1 de T. gondii del kit LSI VetMAX.

\section{Resultados}

En este estudio las 250 muestras de carne de cerdo recolectadas en mercados municipales de la Ciudad de Guatemala, procesadas con la prueba de qPCR fueron negativas a T. gondii.

Los resultados negativos pueden deberse a que existe un $68 \%$ de probabilidad que las muestras provengan de granjas tecnificadas, que cumplen las normativas de bioseguridad y siguen el proceso de trazabilidad, al ser los animales faenados en rastros industriales certificados para luego ser trasladados a los mercados municipales (Dirección de Sanidad Animal del VISAR-MAGA, 2020). Se puede inferir que los esfuerzos de bioseguridad y trazabilidad de las granjas tecnificadas bajo la guía de los servicios veterinarios oficiales han tenido efecto positivo en la porcinocultura, lo que se ve reflejado en la ausencia de T. gondii en la carne de cerdo muestreada.

Sin embargo, el estudio no es capaz de desestimar a la carne de cerdo de los mercados municipales como potencial fuente de infección de T. gondii; ya que según la Dirección de Sanidad Animal del VISAR-MAGA (2020) en mercados locales y regionales, en los que se encuentran los mercados municipales, un $32 \%$ de la carne de cerdo es proveniente de las granjas semi tecnificadas. Dichas granjas se caracterizan por alimentar a los cerdos con desperdicios y permitirles libre pastoreo, ambos factores hacen vulnerables a los cerdos a la infección de T. gondii por el ciclo epidemiológico del protozoo (Despommier et al., 2017; Dubey et al., 2020; Hill y Dubey, 2002). 
Aunado a lo anterior, no existe trazabilidad de las granjas semi tecnificada a los puestos de venta, ya que los rastros utilizados pueden ser los municipales o se realiza el faenado en casa de habitación, ninguna de las dos opciones posee certificación y supervisión de los servicios veterinarios oficiales (Dirección de Sanidad Animal del VISAR-MAGA, 2020).

También se debe contemplar que, a pesar de la bioseguridad, las granjas porcinas tecnificas en ocasiones no logran ser $100 \%$ libres de infecciones parasitarias, como es el caso de T. gondii. Esto se demuestra en el estudio de Carranza C y colaboradores (2016) en Perú, donde por prueba de inmunofluorescencia indirecta (IFI) se comprobó la infección por este agente parasitario en 240 cerdos de granjas tecnificadas, equivalente al $21.7 \%$ de positividad. Esto refuerza nuestra deducción que a pesar de los resultados negativos existe la posibilidad que carne de cerdo infectada con quistes de T. gondii llegue al consumidor final en los mercados municipales a pesar de las prácticas de crianza, matanza y comercialización.

\section{Discusión}

Como demuestra el estudio en Perú, la investigación en la cadena de producción es de suma importancia para conocer el potencial de riesgo real que la carne de cerdo puede ser para la infección de T. gondii; así como formular conclusiones certeras con los resultados negativos. Un ejemplo de ello es el de Galván-Ramírez y colaboradores (2010) que utilizando PCR en 48 muestras de carne de cerdo provenientes de carnicerías en Ocotlán, Jalisco, México obtuvo resultados totales negativos.

El autor señala que fue un resultado contradictorio a las tendencias que en el mismo país se han visto para la carne de cerdo. Dichas tendencias se respaldan en estudios como el realizado por Jimenez-Coello y colaboradores (2013) que realizó enzimoinmunoanálisis de adsorción (ELISA) IgG indirecta en 30 cerdos de traspatio de una zona endémica tropical de México obteniendo un $50 \%$ de resultados positivos.

Este estudio es pionero en el intento por descubrir una posible fuente de infección de T. gondii en Guatemala. No existen otros estudios en las fuentes de infección de $T$. gondii en ninguna de las partes de la cadena de producción de carne de cerdo. Nuestros resultados nos hacen concluir que se deben desarrollar más estudios de la cadena epidemiológica de la to- 
xoplasmosis en cerdos, así como de las diferentes etapas de la cadena productiva de la carne de cerdo, porque existen fuentes de infección que pueden presentarse desde la crianza de los animales hasta los hábitos del consumidor final que tiende a comer carne cruda o insuficientemente cocida. Por lo anterior, no descartamos que el riesgo de infección de toxoplasmosis siga presente en nuestro medio asociado al consumo de carne de cerdo.

\section{Agradecimientos}

La presente investigación ha sido realizada gracias al apoyo de la Facultad de Medicina Veterinaria y Zootecnia, al Instituto de Investigaciones en Ciencia Animal y Ecosalud, a la Gremial de Técnicos Especialistas en Cerdos de Guatemala por el aval y al financiamiento aportado por la Dirección General de Investigación de la Universidad de San Carlos de Guatemala. Esta investigación fue cofinanciada por Digi-Usac 2019, proyecto: 4.8.63.1.90.

\section{Referencias}

Acha, P., Szyfres, B. (2003). Zoonosis y enfermedades transmisibles comunes al hombre y a los animales ( $3^{\mathrm{a}} \mathrm{ed}$, Vol. 1). https://www.paho.org/ hq/dmdocuments/2017/Acha-Zoonosis-Spa.pdf

Almeria, S. y Dubey, J.P. (2020). Foodborne transmission of Toxoplasma gondii infection in the last decade. An overview. Research in Veterinary Science, 135, 371-385. https://doi.org/10.1016/j.rvsc.2020.10.019

Carranza C, F., Suárez A, F., Chávez V, A. y Casas A, E. (2016). Frecuencia de toxoplasmosis en cerdos provenientes de granjas tecnificadas y el sexo como factor de riesgo. Revista de investigaciones veterinarias del Perú, 27(4), 799-804. http://dx.doi.org/10.15381/rivep.v27i4.12565

Despommier, D.D., Griffin, D.O., Gwads, R.W. y Knirsch, C.A. (2017). Parasitic diseases. (6.a. ed.). Parasites Without Borders, Inc. NY. https://www.researchgate.net/profile/Dickson-Despommier/publication/316439090_Parasitic_Diseases_6th_Edition_2nd_Printing_2017/ 
links/58fe28d245851565029de006/Parasitic-Diseases-6th-Edition-2nd-Printing-2017.pdf

Dirección de Sanidad Animal del Viceministerio de Sanidad Agropecuaria y regulaciones del Ministerio de Agricultura, Ganadería y Alimentación. (2020). Caracterización Nacional Porcicultura de Guatemala [Documento PDF]. Viciministerio de Sanidad Agropecuaria y regulaciones del Ministerio de Agricultura, Ganadería y Alimentación. https://visar.maga. gob.gt/visar/2019/sa/caract-porc.pdf

Dubey, J. P. (2010). Toxoplasmosis of animals and humans ( $2^{\mathrm{a}}$ ed). CRC Press.

Dubey, J.P., Cerqueira-Cezar, C.K., Murata, F.H.A., Kwok, O.C.H., Hill, D., Yang, Y.R. y Su, C. (2020). All about Toxoplasma gondii infections in pigs: 2009-2020. Veterinary Parasitology, 288, 109-185. https://doi.org/10.1016/j.vetpar.2020.109185

Galván-Ramírez, M. L., Madriz Elisondo, A. L., Rico Torres, C. P., Luna-Pastén, H., Rodríguez Pérez, L. R., Rincón-Sánchez, A. R., Franco, R., Salazar-Montes, A. y Correa, D. (2010). Frequency of Toxoplasma gondii in pork meat in Ocotlán, Jalisco, México. Journal of Food Protection, 73(6), 1121-1123. https://doi.org/10.4315/0362-028X-73.6.1121

Gibson, C.L. y Coleman, N. (1958). The prevalence of Toxoplasma antibodies in Guatemala and Costa Rica. The american journal of tropical medicine and hygiene, 7(3), 334-338. https://doi.org/10.4269/ajtmh.1958.7.334

Hill, D. y Dubey, J. (2002). Toxoplasma gondii: transmission, diagnosis and prevention. Clinical Microbiology and Infection, 8(10), 634-640. https:// doi.org/10.1046/j.1469-0691.2002.00485.x

Jimenez-Coello, M., Acosta-Vianaa, K.Y., Guzman-Marina, E., Gutierrez-Ruiz, E.J. y Rodriguez-Vivas, R.I., Bolio-González, M.E., Ortega-Pacheco, A. (2013). Presencia de anticuerpos de Toxoplasma gondii en cerdos y gatos de traspatio en una región endémica tropical de México. Tropical and subtropical agroecosystems, 16(1), 89-92. https://www.redalyc.org/ articulo.oa?id=93927469013 
Jones, J. L. y Dubey, J. P. (2012). Foodborne toxoplasmosis. Clinical Infectious Diseases, 55(6), 845-851. https://doi.org/10.1093/cid/cis508

Jones, J. L., López, B., Álvarez Mury, M., Wilson, M., Klein, R., Luby, S., y Maguire, J. H. (2005). Toxoplasma gondii infection in rural guatemalan children. The American Journal of TropicalMmedicine and Hygiene, 72(3), 295-300. https://doi.org/10.4269/ajtmh.2005.72.295

López, B., Jones, J. y Arana, B. (2008). Toxoplasmosis ocular en niños de Guatemala. Revista de la Universidad del Valle de Guatemala, 1(17), 80-89. https://www.uvg.edu.gt/descargables/revista-uvg/Revista\%2017_.pdf

Lora, F., Aricapa, H. J., Pérez, J. E., Arias, L. E., Idarraga, S. E., Mier, D. y Gómez, J. (2007). Detección de Toxoplasma gondii en carnes de consumo humano por la técnica de reacción en cadena de la polimerasa en tres ciudades del eje cafetalero. Infectio, 11(3), 117-123. http://www. scielo.org.co/pdf/inf/v11n3/v11n3a04.pdf

Sánchez Artigas, R., Cobos Valdés, D., Sánchez Cruz, L., Miranda Cruz, L., Camejo Roviralta, L. y Araujo Baptistal, L. (2016). La toxoplasmosis observada como un problema no resuelto. Revista Cubana de Investigaciones Biomédicas, 36(3), 272-283. http://scielo.sld.cu/scielo. php?script=sci_arttext\&pid=S0864-03002016000300006

Sinibaldi, J. y De Ramirez, I. (1992). Incidence of congenital toxoplasmosis in live Guatemalan newborns. European journal of epidemiology, 8, 516520. https://doi.org/10.1007/BF00146369

\section{Sobre las autoras}

\section{Flor Dinorah Porras}

Médica veterinaria, Facultad de Medicina Veterinaria y Zootecnia, con Maestría en gestión de la calidad con especialización en inocuidad de los alimentos de la Facultad de Ciencias Químicas y Farmacia, de la Universidad de San Carlos de Guatemala. Coordinadora de la investigación presentada, que fue cofinanciada por Digi-Usac 2019, proyecto: 4.8.63.1.90. 


\section{Ana Isabel Girón Samayoa}

Estudiante con cierre de pénsum de la licenciatura de Medicina Veterinaria, en la Universidad San Carlos de Guatemala. Actual estudiante de segundo año de la licenciatura en educación con especialización en ciencias químicas y biológicas de la Universidad del Valle de Guatemala. Auxiliar de investigación para la investigación presentada, que fue cofinanciada por Digi-Usac 2019, proyecto: 4.8.63.1.90.

\section{Copyright (c) Flor Dinorah Porras y Ana Isabel Girón Samayoa}

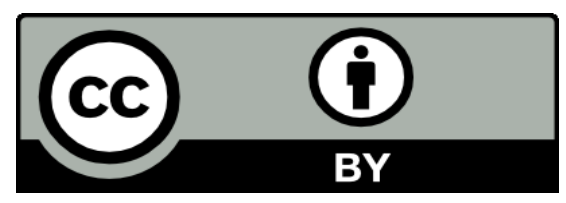

Este texto está protegido por una licencia CreativeCommons 4.0.

Usted es libre para compartir, copiar y redistribuir el material en cualquier medio o formato y adaptar el documento, remezclar, transformar y crear a partir del material para cualquier propósito, incluso comercialmente, siempre que cumpla la condición de atribución: usted debe reconocer el crédito de una obra de manera adecuada, proporcionar un enlace a la licencia, e indicar si se han realizado cambios. Puede hacerlo en cualquier forma razonable, pero no de forma tal que sugiera que tiene el apoyo del licenciante o lo recibe por el uso que hace. 